\title{
NEW SPECIES OF FRESHWATER SNAILS OF STENOTHYRA (STENOTHYRIDAE - MESOGASTROPODA) FROM VIETNAM
}

\author{
DANG NGOC THANH, HO THANH HAI
}

\section{Institute of Ecology and Biological Resources}

Stenothyridae P. Fischer, 1887 is a family of snails with shell small or very small. Species of this family live in fresh or brackish waters, and distributed in Asia, islands of western Pacific and Australia. 6 species of the Stenothyra genus were recorded in Vietnam including Stenothyra messageri Bavey et Dautzenberg, 1900, S. monilifera Benson, 1856, S. polita (A. Adams, 1851), S. divalis (Gould), S. cyrtochila van Benthem, 1959, and S.. schlickumi Brandt, 1968. Among them, Stenothyra messageri distributed only in freshwater bodies in northern Vietnam. Up to now, this species is an endemic species of Vietnam. The remains largely distribute in both fresh water and brackish water of estuarine area, mangrove forest.

Among samples of snail recently collected from the Cai Lon river (a tributary of Mekong river) located in Vinh Loc commune, Kiengiang province, southern Vietnam, and from river mouth of the Red river at Giao Thuy district, Namdinh province, northern Vietnam, some specimens of the Stenothyridae family are found. Analyzed results of snail samples show that this is three new species belonging to the unique genus Stenothyra Benson of this family. The following are descriptions of these new species of snail.

\section{Stenothyra ovata Dang et Ho, sp. nov.}

Holotype: Length $6.2 \mathrm{~mm}$, breadth $4.2 \mathrm{~mm}$, length of aperture $1.9 \mathrm{~mm}$, breadth of aperture $2.1 \mathrm{~mm}$, length of the body whorl $5.3 \mathrm{~mm}$, collected from the Cai Lon river - a tributary of Mekong river, Kien Giang province, southern Vietnam. Paratype: 2 specimens, collected also from above location, type specimens deposited in IEBR, Reference collection, Hanoi, Vietnam.

Diagnosis: Shell rather large for the genus, ovate-conic in shape, with obtuse apex, eroded. Shell solid but translucent, colour of shell changed from yellowish-brown to rusty-brown. Whorls 3-4, the body whorl distinctly compressed dorso-ventrally. Whorls sculptured with regular spiral lines of distinct pits. The basal keel of outer margin of peristome developed forming a beak-like with round top.

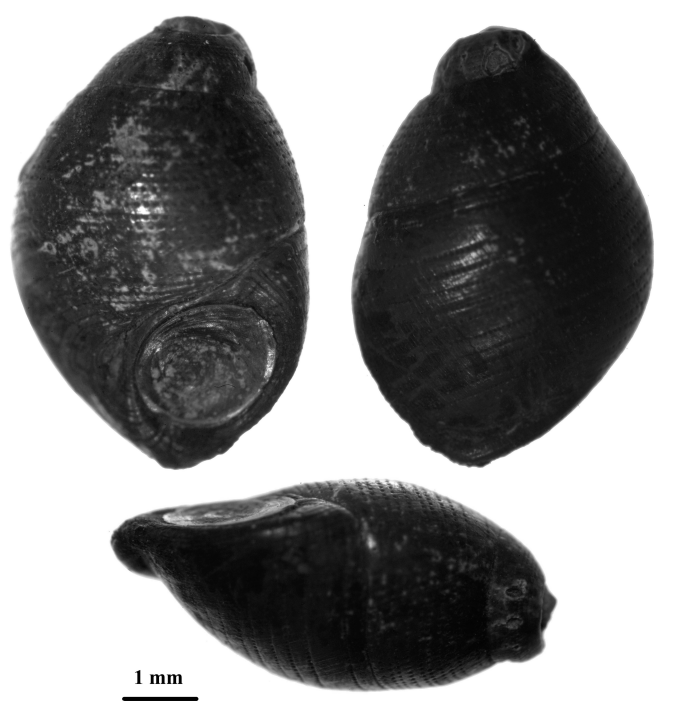

Figure 1. Holotype of Stenothyra ovata Dang et Ho, sp. nov.

Description: Shell rather large for the genus, ovate-conic in shape, with obtuse apex, eroded. Length of shell reaching to over $6 \mathrm{~mm}$. Spire eroded with 3-4 remaining whorls. Body whorl distinctly compressed dorso-ventrally, breadth 4.0-4.2 mm, length of body whorl reaching to $4.8-5.3 \mathrm{~mm}$, longer than spire length, equal $4 / 5$ length of shell. Suture shallow. Shell whorl sculptured with pitted spiral lines, about 10 lines on the penultimate whorl, 12 lines on the body whorl between suture and periphery, about 8 lines on the base of peristome, nearly to umbilicus. Shell solid but 
translucent, colour of shell changed from yellowish-brown to rusty-brown.

End part of the last whorl strongly constricted and forming a flat plane, with the aperture in which placed. Aperture nearly oval. View from back shell, the body whorl constricted and forming a keel in basal underneath. View from front of shell, the basal keel of lower outer margin of peristome developed and forming a beak-like with round top. Umbilicus distinct in form of narrow groove. Operculum oval, glossy, corneous, translucent, paucispiral with sub-central nucleolus.

Size (mm): H: 6.0-6.2; 1: 4.0-4.2; V: 4.8-5.3; Lo: 1.6-2.0; lo: 2.1-2.2.

Habitat: Fresh water to brackish water, this species living in mud-flats at littoral areas of tributaries, channels nearly river mouth water.

Remarks: The new species Stenothyra ovata sp. nov. has some characteristics that are similar to Stenothyra polita (Adams, 1851) and S. prasonga Brandt, 1974 (a species only found in Thailand-Brandt, 1974) such as rather large size, 6-over $7 \mathrm{~mm}$; Shell rather thick, solid but translucent. Whorls are sculptured with moderately regular spiral lines of distinct pits; especially structure location of aperture. Peristome thick, continuous, formed by the constricted end part of the last whorl. The basal keel of lower outer margin of peristome developed and forming a beak-like with round top. Living habitats of these species are smooth fresh-brackish waters nearly river mouth.

Beside similar characteristic mentioned as above, this new species clearly differs from its closest relative species in some basal features presented in below table.

Etymology: The new species name is conformed to the ovate shape of the shell of this species.

Table 1

Different characteristics of shell between Stenothyra ovata sp. nov. and its closest relatives, Stenothyra polita (Adams), S. prasonga Brandt

\begin{tabular}{|l|l|l|l|}
\hline $\begin{array}{c}\text { Morphological } \\
\text { characteristics of shell }\end{array}$ & \multicolumn{1}{|c|}{$\begin{array}{c}\text { Stenothyra ovata sp. } \\
\text { nov. }\end{array}$} & \multicolumn{1}{|c|}{ Stenothyra polita } & \multicolumn{1}{c|}{$\begin{array}{c}\text { Stenothyra } \\
\text { prasongi }\end{array}$} \\
\hline Shape & Ovate & $\begin{array}{l}\text { Almost triangular } \\
\text { shape }\end{array}$ & $\begin{array}{l}\text { Almost triangular } \\
\text { shape }\end{array}$ \\
\hline Apex & Obtuse & Pointed & rather obtuse \\
\hline Number of whorl & \multicolumn{1}{|c|}{$3-4$} & \multicolumn{1}{c|}{5} \\
\hline Whorls & Moderately flatted & Convex & Convex \\
\hline Suture & Shallow & Deep & Deep \\
\hline Pitted spiral lines & Distinct, deep & Translucent, shallow & Distinct, deep \\
\hline Colour of shell & Yellowish-brown to & $\begin{array}{l}\text { Grey with black } \\
\text { pigment and white } \\
\text { patches }\end{array}$ & $\begin{array}{l}\text { Rusty-brown } \\
\text { patches }\end{array}$ \\
\hline $\begin{array}{l}\text { Ratio of body whorl } \\
\text { length/length of shell }\end{array}$ & \multicolumn{1}{|c|}{$3 / 5$} & \multicolumn{1}{|c|}{$3 / 5$} \\
\hline
\end{tabular}

\section{Stenothyra conica Dang et Ho, sp. nov.}

Holotype: length $2.6 \mathrm{~mm}$, breadth $1.4 \mathrm{~mm}$, length of aperture $0.6 \mathrm{~mm}$, breadth of aperture $0.9 \mathrm{~mm}$, length of the body whorl $2.0 \mathrm{~mm}$, collected from the Cai Lon river, tributary of Mekong river, Kiengiang province, southern Vietnam. Paratype: 1 specimen, type specimens collected also from above location, deposited in IEBR, Reference collection, Hanoi, Vietnam.
Diagnosis: Shell very small for the genus, moderately conic in shape, with obtuse apex. Spire short, equal $1 / 4$ of shell. Whorls $3-4$, the last whorl largely convex, moderately compressed dorso-ventrally. Shell thin, glossy, transparent, smooth with dimly oblique lines. Colour of shell changed from light yellow to brown yellow. Aperture ovoid, peristome sharp angled right above. 


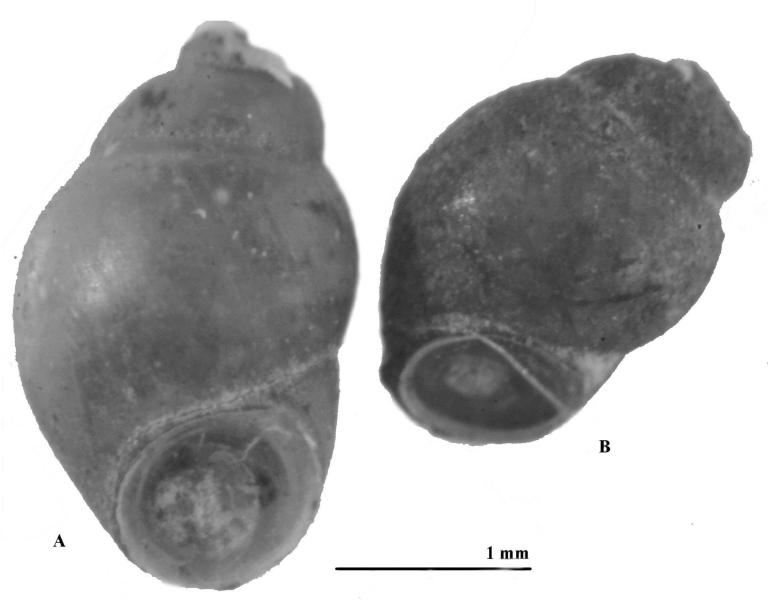

Figure 2. Stenothyra conica Dang et Ho, sp. nov. (A: Holotype; B: Paratype)

Description: Shell rather small for the genus, moderately conic in shape, with obtuse apex. Length of shell reaching to $2.6 \mathrm{~mm}$. Spire short, equal 1/4 of shell. Spire with 3-4 whorls, suture rather deep, distinct. Whorls convex, the body whorl strongly convex, breadth $1.4 \mathrm{~mm}$, length of body whorl reaching to $2.0 \mathrm{~mm}$, longer than spire length, equal 3/4 length of shell. Shell smooth with dimly oblique lines and pigmental dots, thin, transparent, colour of shell changed from light yellow to brown yellow. Aperture ovoid, peristome continuous no thicked, forming an angle right upper.

Umbilicus distinctly narrow groove in form. Operculum ovoid, thin, paucispiral with subcentral nucleous.

Size (mm): H: 2.1-2.6; 1: 1.2-1.4; V: 1.7-2.0; Lo: 0.5-0.6; lo: 0.8-1.0.

Habitat: Fresh water to brackish water, this species living in mud-flats at littoral areas of tribuataries, channels nearly river mouth water.

Remarks: This new species clearly differs from various species of the genus distributed in fresh and brackish water nearly river mouth area in small size, conic in shape, aperture ovoid angled right above. Shell thin, colour of shell changed from light yellow to brown yellow. Spire short equal 1/4 length of shell.

Etymology: The new species name is conforming to the conic shape of the shell.

Table 2

Different characteristics of shell between Stenothyra conica sp. nov. and its closest relatives, Stenothyra monilifera and $S$. divalis

\begin{tabular}{|l|l|l|l|}
\hline $\begin{array}{c}\text { Morphological } \\
\text { characteristis of shell }\end{array}$ & $\begin{array}{l}\text { Stenothyra conica } \text { sp. } \\
\text { nov. }\end{array}$ & \multicolumn{1}{|c|}{$\begin{array}{c}\text { Stenothyra } \\
\text { monilifera }\end{array}$} & \multicolumn{1}{|c|}{ Stenothyra divalis } \\
\hline Shape & $\begin{array}{l}\text { Conic, thin, } \\
\text { transparent }\end{array}$ & $\begin{array}{l}\text { Elongately ovate, } \\
\text { translucent }\end{array}$ & $\begin{array}{l}\text { Ovate, rather thick, } \\
\text { translucent }\end{array}$ \\
\hline Aperture & Ovoid & Elliptic & Elliptic \\
\hline Sculpture & $\begin{array}{l}\text { Smooth, dimly oblique } \\
\text { lines }\end{array}$ & Pitted spiral lines & $\begin{array}{l}\text { Smooth, distinctly } \\
\text { oblique lines }\end{array}$ \\
\hline Size & $\begin{array}{l}\text { Rather small for the } \\
\text { genus (H: } 2.1-2.6 \mathrm{~mm})\end{array}$ & $\begin{array}{l}\text { Medium for the genus } \\
\text { (H: 4.0-4.2 mm) }\end{array}$ & $\begin{array}{l}\text { Rather large for the } \\
\text { genus (H: } 4.1-6.2 \mathrm{~mm})\end{array}$ \\
\hline Number of whorl & \multicolumn{1}{|c|}{$3-4$} & 4 & 4 \\
\hline Colour & $\begin{array}{l}\text { Light yellow to brown } \\
\text { yellow }\end{array}$ & $\begin{array}{l}\text { Yellow brown to } \\
\text { black green }\end{array}$ & Yellow brown \\
\hline $\begin{array}{l}\text { Ratio of body whorl } \\
\text { length/length of shell }\end{array}$ & \multicolumn{1}{|c|}{$3 / 4$} & $3 / 4$ & $2 / 3-3 / 4$ \\
\hline
\end{tabular}

\section{Stenothyra alba Dang et Ho, sp. nov.}

Holotype: length $2.5 \mathrm{~mm}$, breadth $1.5 \mathrm{~mm}$, length of aperture $0.5 \mathrm{~mm}$, breadth of aperture $0.7 \mathrm{~mm}$, length of the body whorl $1.9 \mathrm{~mm}$, collected from the Ba Lat river mouth belonging to the Hong river at Giao Thuy district,
Namdinh province, northern Vietnam. Paratype: 6 specimens, collected also from above location, deposited in IEBR, Reference collection, Hanoi, Vietnam.

Diagnosis: Shell rather small for the genus, moderately triangular in shape, with rather 
obtuse apex. Shell solid but translucent, smooth, colour of shell changed from white to milky with some brown yellow dot. Whorls 4 .

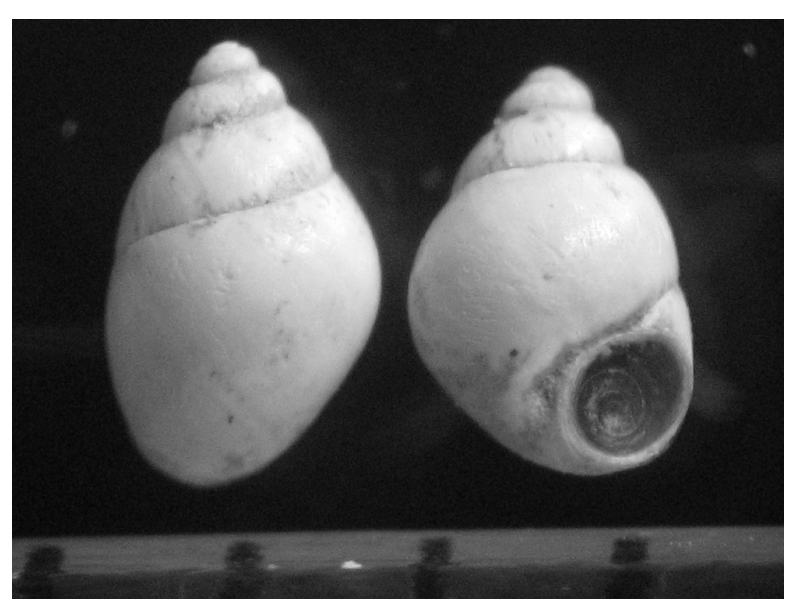

Figure 3. Holotype of Stenothyra alba Dang et Ho, sp. nov.

Description: Shell rather small for the genus, nearly conical in shape, with rather obtuse apex. Length of shell reaching to over 2 $\mathrm{mm}$. Spire with 4 whorls, suture rather deep, distinct. Body whorl largely convex, breadth $0.7-0.8 \mathrm{~mm}$, length of body whorl reached to 1.4 $\mathrm{mm}$, longer than spire length, equal $3 / 5$ length of shell. Shell smooth, no rib, ridge. Shell solid but translucent, smooth, colour of shell changed from white to milky with some brown yellow dot.

Aperture nearly ovoid, peristome thick continuous, formed by the constricted end part of the last whorl. Umbilicus in form distinctly deep groove, transverse. Operculum ovoid, rather round, paucispiral with excentral nucleous.

Size (mm): H: 2.0-2.5; 1: 1.1-1.5; V: 1.5-1.9; Lo: $0.35-0.5$; lo: 0.5-0.7.

Habitat: Brackish water creeks in mud-flats and mangrove forest.

Remarks: The new species Stenothyra alba sp. nov. has characteristics that are similar to species distributed in brackish water such as shell thick, solid, peristome thick, continuous. However, this new species clearly differs from all known species belonging to genus Stenothyra in both Vietnam and Thailand, such as small in size, shell smooth, colour of shell milk white with some brown yellow dot.

Etymology: The new species name is referred to the white colour of the shell.

Comparison of shell characteristics of Stenothyra alba sp. nov. and its closest relatives, Stenothyra polita and Stenothyra cyrtochila

\begin{tabular}{|c|c|c|c|}
\hline $\begin{array}{c}\text { Morphological } \\
\text { characteristics of shell }\end{array}$ & $\begin{array}{c}\text { Stenothyra alba sp. } \\
\text { nov. }\end{array}$ & Stenothyra polita & $\begin{array}{c}\text { Stenothyra } \\
\text { cyrtochila }\end{array}$ \\
\hline Shape & $\begin{array}{l}\text { Almost triagualar } \\
\text { shape, shell thick, } \\
\text { solid }\end{array}$ & $\begin{array}{l}\text { Almost triangular } \\
\text { shape, shell thick, } \\
\text { solid }\end{array}$ & Ovate, shell think \\
\hline Size & $\begin{array}{l}\text { Rather small for the } \\
\text { genus } \\
\text { (H: } 2.0-2.5 \mathrm{~mm})\end{array}$ & $\begin{array}{l}\text { Rather large for the } \\
\text { genus } \\
\text { (H: } 5.9-7.2 \mathrm{~mm})\end{array}$ & $\begin{array}{l}\text { Rather small for the } \\
\text { genus } \\
\text { (H: } 1.8-2.4 \mathrm{~mm})\end{array}$ \\
\hline Apex & Rather obtuse & Pointed & Obtuse \\
\hline Number of whorl & 4 & 5 & 3,5 \\
\hline Whorls & Convex & Convex & Rather convex \\
\hline Sucture & Deep & Deep & Shallow \\
\hline Spiral line & Absent & $\begin{array}{l}\text { Translucent, with } \\
\text { shallowly pitted }\end{array}$ & $\begin{array}{l}\text { Distinct, with deep- } \\
\text { brown colour }\end{array}$ \\
\hline Colour of shell & $\begin{array}{l}\text { Milk-white with } \\
\text { some brown yellow } \\
\text { dot }\end{array}$ & $\begin{array}{l}\text { Grey with black } \\
\text { pigment and white } \\
\text { patches }\end{array}$ & $\begin{array}{l}\text { Reddish brown, } \\
\text { bronze }\end{array}$ \\
\hline $\begin{array}{l}\text { Ratio of body whorl } \\
\text { length/length of shell }\end{array}$ & $3 / 5$ & $3 / 5$ & $2 / 3-3 / 4$ \\
\hline
\end{tabular}


REFERENCES

1. Brandt R. A. M., 1974: The non-marine aquatic Mollusks of Thailand. Frankfurt am Main, 423 pages.

2. George M. Davis, Cui-E Chen, Xin-Guo Xing, Chun Wu, 1988: The Stenothyra of
China. No.2: Stenothyra hunanensis. Proceeding of the Academy Natural Sciences of Philadelphia 140(2): 247-266.

3. Yen T. C., 1939: Naturf. Ges., 444: 1-234, 16 pls.

4. Yih-Tsong Ueng, Jiang-Ping Wang, 2003:

J. Taiwan Museum, 27: 23-40.

\title{
BA LOÀI ỐC NƯớC NGOTT MỚI THUỘC GIỐNG STENOTHYRA (STENOTHYRIDAE - MESOGASTROPODA) Ở VIÊTT NAM
}

\author{
ĐặNG NGỌC THANH, HỒ THANH HẢI
}

\section{TÓM TÁT}

Thời gian qua, trong số vật mẫu thu được ở một số địa điểm như ở sông Cái Lớn ở vùng đồng bằng thấp gần biển thuộc địa phận tỉnh Kiên Giang, Nam Bộ Việt Nam, ở vùng cửa sông Hồng tại Giao Thuỷ, tỉnh Nam Định, có một số vật mẫu thuộc họ ốc Stenothyridae. Các kết quả phân tích vật mẫu đã xác định đây là 3 loài mới thuộc 1 giống duy nhất Stenothyra của họ ốc này. Sau đây là mô tả các loài ốc mới đó.

\section{Stenothyra ovata Dang et Ho, sp. nov. (hình 1)}

Holotype: 1 mẫu vật (H: 6,2 mm; 1:4,2; V; 5,3; Lo:1,9; lo2,1), Paratyp: 2 mẫu vật, thu được tại sông Cái Lớn thuộc hệ thống sông Mekong ở tỉnh Kiên Giang, Nam Bộ Việt Nam, cách vùng cửa sông tại TP. Rạch Giá khoảng 30 km. Lưu giữ tại Bộ sưu tập, Viện Sinh thái và Tài nguyên sinh vật, Hà Nội, Việt Nam.

Chẩn loại: Vỏ kích thước lớn, dày, chắc, đỉnh tày, tháp ốc rất ngắn, chỉ bằng $1 / 5$ chiều cao vỏ, vòng xoắn cuối phình rộng, dẹp theo hướng lưng-bụng. Vỏ gần hình bàu dục, có các đốm màu nâu vàng tới nâu, mặt vỏ có những đường xoắn khá đều đặn với những nốt hõm sâu. Lỗ miệng gần bàu dục, nằm nông, phần gốc mép ngoài vành miệng kéo dài xuống phía dưới thành mấu tròn đầu. Lỗ rốn thành rãnh hẹp, rõ.

Mô tả: ốc có kích thước khá lớn so với các loài trong giống, vỏ gần hình trứng, phình ở giữa, vỏ dày, chắc, giống như sừng, đỉnh tày, bị gặm mòn. Tháp ốc rất ngắn, chỉ dài bằng $1 / 5$ chiều cao vỏ.

Đỉnh vỏ bị gặm mòn, chỉ thấy 3-4 vòng xoắn. Vòng xoắn cuối cao, phình to, dẹp theo lưng-bụng. Chiều cao vòng xoắn cuối tới $4 / 5$ chiều cao vỏ. Mặt vỏ có các đốm màu nâu vàng tới nâu, chỉ có các đường chỉ xoắn với những nốt nhỏ, thấy rõ hơn so với loài Stenothyra polita. Có 10 đường ở vòng xoắn áp cuối, 12 đường ở vòng xoắn cuối, 8-9 đường ngắn ở phần gốc trái vành miệng, gần lỗ rốn. Phần cuối của vòng xoắn cuối bẹp lại thành dạng mặt phẳng, ở gữa là lỗ miệng. Nhìn từ mặt sau vỏ, vòng xoắn cuối thắt lại và tạo thành gờ sống ở phía dưới. Nhìn từ mặt trước, phần gốc mép ngoài vành miệng kéo dài xuống phía dưới thành mỏ tròn đầu.

Lỗ miệng gần bàu dục, mỏ nằm hơi xiên. Vành miệng dày, liên tục. Lỗ rốn hình rãnh hẹp, rõ. Nắp miệng dày, gần tròn, ít vòng xoắn, nhân lệch tâm.

Kích thước: H: 6,0-6,2; 1: 4,0-4,2; V: 4,8-5,3; Lo: 1,6-2,0; lo: 2,1-2,2.

Sinh học, sinh thái: Sống vùi trong bùn, ăn mùn bã hữu cơ mục nát ở ven bờ các sông, lạch nước ngọt, lợ vùng đồng bằng gần biển.

Nhận xét: Loài mới này có những đặc điểm giống với các loài Stenothyra polita và S. prasongi (loài mới thấy ở Thái Lan) ở kích thước lớn 6-trên $7 \mathrm{~mm}$; vỏ dày chắc; mặt vỏ có các đường chỉ xoắn với nốt hõm; đặc biệt cấu tạo và hình thái lỗ miệng, vành quanh miệng ở góc dưới phát triển thành hình mấu tròn đầu, và sinh cảnh sống cũng là vùng nước ngọt, lợ gần cửa sông. Tuy nhiên, giữa hai loài này vẫn có những khác biệt cơ bản. 


\section{Stenothyra conica Dang et Ho, sp. nov. (hình 2)}

Holotype: 1 mẫu vật (H: 2,6; 1: 1,4; V; 2,0; Lo: 0,6; lo 0,9). Paratyp: 1 mẫu vật, thu được tại sông Cái Lớn thuộc hệ thống sông Mekong ở tỉnh Kiên Giang, Nam Bộ Việt Nam, cách vùng cửa sông tại TP. Rạch Giá khoảng 30 km. Lưu giữ tại Bộ sưu tập, Viện Sinh thái và Tài nguyên sinh vật, Hà Nội, Việt Nam.

Chẩn loại: Vỏ có kích thước khá nhỏ, mỏng, trong, dáng hình côn, đỉnh tày, tháp ốc ngắn, chỉ bằng $1 / 4$ chiều cao vỏ. Có 3-4 vòng xoắn, vòng xoắn cuối phình rộng, phần bụng hơi dẹt. Vỏ nhẵn, chỉ có các chấm sắc tố đậm nhỏ và đường xiên mờ. Vỏ có màu vàng nâu, trong. Lỗ miệng hình trứng, hơi tạo thành góc ở trên phải.

Mô tả: ốc có kích thước rất nhỏ so với các loài trong giống, vỏ dáng hình côn, mỏng, trong, đỉnh tày, thường bị gặm mòn. Tháp ốc ngắn, dài bằng $1 / 4$ chiều cao vỏ.

Có 3-4 vòng xoắn, rãnh xoắn sâu, thấy rõ. Vòng xoắn phồng, vòng xoắn cuối phình rộng, phần bụng hơi dẹt, chiều rộng $1,4 \mathrm{~mm}$, chiều cao $2,0 \mathrm{~mm}$ bằng khoảng $3 / 4$ chiều cao vỏ. Mặt vỏ nhẵn, màu vàng nâu sáng, điểm các sắc tố đậm, có các đường xiên mờ.

Lỗ miệng hình trứng, vành miệng sắc, liên tục, hơi tạo thành góc ở phía trên phải. Lỗ rốn thành khe hẹp, rõ. Nắp miệng mỏng, hình trứng, ít vòng xoắn, nhân lệch tâm.

Kích thước: $\mathrm{H}: 2,1-2,6 \mathrm{~mm}$; 1: 1,2-1,4 mm; V: 1,7-2,0 mm; Lo: 0,5-0,6 mm; lo: 0,8-1,0 mm.

Sinh học, sinh thái: Sống vùi trong bùn, ăn mùn bã hữu cơ mục nát ở ven bờ các sông, lạch nước ngọt, lợ vùng đồng bằng gần biển.

Nhận xét: Loài ốc mới này có các nét khác hẳn với những loài khác trong giống cùng phân bố ở vùng nước ngọt, lợ nhạt gần biển ở Việt Nam ở chỗ kích thước rất nhỏ, hình côn, lỗ miệng hình trứng, hơi tạo thành góc trên phải. Vỏ mỏng, màu vàng nâu trong, tháp ốc ngắn chỉ bàng $1 / 4$ chiều cao vỏ.

\section{Stenothyra alba Dang et Ho, sp. nov. (hình 3)}

Holotype: 1 mẫu vật (H: 2,5; 1. 1,5; V; 1,9; Lo: 0,5; lo 0,7). Paratyp: 6 mẫu vật, thu được tại vùng cửa Ba Lạt, thuộc sông Hồng thuộc huyện Giao Thuỷ, tỉnh Nam Định, bắc Việt Nam. Lưu giữ tại Bộ sưu tập, Viện Sinh thái và Tài nguyên sinh vật, Hà Nội, Việt Nam.

Chẩn loại: Vỏ kích thước nhỏ, dày, chắc, đỉnh hơi tày, tháp ốc ngắn, chỉ bằng $2 / 5$ chiều cao vỏ. Có 4 vòng xoắn, vòng xoắn cuối phình rộng. Vỏ gần hình nón, nhẵn, không có đường, khía. Vỏ có màu trắng đục với ít điểm sắc tố vàng. Lỗ miệng gần bàu dục, hơi tròn, nằm nông. Vành miệng dày, liên tục. Lỗ rốn thành rãnh hẹp, rõ.

Mô tả: ốc có kích thước khá nhỏ so với các loài trong giống, vỏ gần hình nón, vỏ dày, chắc, đỉnh hơi tày. Tháp ốc ngắn, dài bằng $2 / 5$ chiều cao vỏ.

Có 4 vòng xoắn, rãnh xoắn sâu, thấy rõ. Vòng xoắn cuối phình rộng, chiều rộng $0,7-0,8 \mathrm{~mm}$, chiều cao $1,4 \mathrm{~mm}$ bằng khoảng $3 / 5$ chiều cao vỏ. Mặt vỏ màu trắng đục với ít điểm sắc tố vàng bẩn, mặt vỏ nhẵn, không có đường, khía.

Lỗ miệng hình trứng, hơi tròn, nằm nông, vành miệng dày, liên tục. Lỗ rốn thành khe sâu, rõ, thẳng ngang. Nắp miệng dày, hình trứng, ít vòng xoắn, nhân lệch tâm.

Kích thước: H: 2,0-2,5 mm; 1: 1,1-1,5 mm; V: 1,5-1,9 mm; Lo: 0,35-0,5 mm; lo: 0,5-0,7 mm.

Sinh học, sinh thái: Sống vùi trong bùn, ăn mùn bã hữu cơ mục nát ở các kênh, lạch nước lợ vùng đồng bằng ven biển.

Nhận xét: Loài ốc Stenothyra alba sp. nov. có những đặc điểm hình thái giống với các loài ốc sống ở vùng nước lợ cửa sông như vỏ dày, chắc, vành miệng dày, liên tục. Tuy nhiên, loài mới này có những nét khác hẳn với các loài khác đã biết trong giống Stenothyra nói chung ở Việt Nam, Thái Lan, cũng như ở vùng cửa sông Ba Lạt nói riêng: kích thước nhỏ, vỏ nhẵn, màu trắng đục. 